\title{
An examination of practice effects in recognition
}

\author{
LEO POSTMAN \\ University of California, Berkeley, California 94720
}

\begin{abstract}
A study of practice effects in recognition performance is reported. In each of two experiments, different conditions of training were followed by a critical final test. In Experiment 1, a yes-no procedure was used on the critical test. During the training phase, some subjects were tested by the same method, either with or without item-by-item feedback, whereas others were given forced-choice tests. No significant changes in recognition accuracy were observed, either during the training trials or on the final test. However, feedback and experience with forcedchoice tests both served to increase the bias toward positive responding. In Experiment 2, the forced-choice procedure was used on the critical final test. Either forced-choice tests or yes-no tests were given on the training lists. Again, there were no significant changes in performance. The absence of practice effects is attributed to the difficulty of identifying and implementing test-appropriate strategies.
\end{abstract}

This study addressed two related questions: (1) Does subjects' ability to discriminate between targets and distractors on recognition tests change significantly as a function of practice? (2) Can response biases, if not the accuracy of recognition, be brought under experimental control by systematic manipulations of the conditions of training?

The literature on learning to learn (LTL) provides ample documentation of practice gains in the performance of tasks requiring recall of the target items (ef. Postman, 1971). While LTL in recognition has not been investigated extensively, little or no change in accuracy over successive study-test cycles was found in several experiments, both for categorized lists (Carey \& Lockhart, 1973; Connor, 1977; Jacoby, 1973) and for lists of unrelated words (Connor, 1977; McCormack \& Swenson, 1972). Divergent effects of practice on performance may represent a potentially important difference between recall and recognition. The reasons for such a difference invite careful consideration.

One possible explanation is that encoding and retrieval operations conducive to gains on subsequent tests can be more readily identified during the performance of recall tasks than during the performance of recognition tasks. For example, attention to the development of higher order units can lead to substantial increases in free recall (Mayhew, 1967). More generally, subjects can choose and practice the procedures they find useful for recall. The opportunities to do so with respect to recognition are likely to be much more limited. To be

This research was supported by a grant from the National Science Foundation. I thank Elizabeth Kruesi for her assistance. Requests for reprints should be addressed to Leo Postman, Institute of Human Learning, University of California, Berkeley, California 94720. sure, subjects may learn to concentrate on individual items rather than on the development of subjective groupings, but such a strategy would not necessarily increase their chances of success on recognition tests. As Connor (1977, p. 318) pointed out, subjects do not have precise information about the distractors from which they will have to discriminate the targets. Encoding cannot be focused sharply on those features of the input items that are critical for the required discriminations. Consequently, there may be little change in sensitivity from one test to the next. However, other characteristics of recognition performance that are more directly under the subject's control, in particular response biases, could be modified through experience. This possibility is examined in the present study.

A further question that arises is whether on tests of recognition the benefits of LTL are masked by interlist interference. This possibility exists for recall tasks as well, but the advantages of prior practice are usually sufficient to outweigh any interference effects. However, it is conceivable, at least a priori, that recognition is peculiarly sensitive to the buildup of interference across successive tasks. It is known that recognition accuracy declines as the subject proceeds through the test, presumably because of the interference resulting from exposure to the distractors (cf. Schulman, 1971; Underwood, 1971). In the study of McCormack and Swenson (1972), there was some indication that such interference may carry over from one list to the next. However, the increases in false alarm rates across lists were quite small. Thus far, there appears to be little empirical support for an assumption that LTL and interlist interference balance each other. Special attention is given to this issue in the analysis of the experimental results reported below.

Finally it is possible that the information required for 
successful discrimination between targets and distractors is stored obligatorily or automatically. If so, no practice effects would be expected. Such an interpretation would be in line with the conclusions of Hasher and Chromiak (1977) regarding the process of frequency discrimination. These investigators found that young children and college students did not differ in the accuracy of their frequency judgments and that adults did not show an improvement as a function of practice, regardless of whether they were given feedback about the correctness of their choices. In light of these findings, Hasher and Chromiak advanced the hypothesis that frequency tagging occurs automatically when incoming information is processed. They also pointed out that recognition has been considered a special case of frequency discrimination (Underwood, 1971). On this assumption, the hypothesis of automatic encoding would appear to be directly applicable to recognition. In this connection, it is interesting to note that in a recent study of children's memory, no improvement in the accuracy of word recognition was found as a function of age (Naus, Ornstein, \& Kresthool, 1977).

We have considered alternative explanations for the failure of recognition performance to improve with practice. The existing evidence for the absence of LTL effects, however, is still quite limited. In several of the experiments mentioned earlier, categorized lists were used, with targets and distractors drawn from the same taxonomic classes. Such materials represent a special case; discrimination between instances of the same category is intrinsically difficult and may be relatively insensitive to practice effects. In the experiments with noncategorized materials, the lists were relatively short and the initial level of recognition was high; thus, there was limited opportunity for improvement. Given the limitations of the available data and the need for caution in the interpretation of null results, a further systematic examination of LTL in recognition appeared to be useful.

In order to focus on the measurement of test-specific skills, the logic of transfer designs was extended to the analysis of LTL effects. With all groups receiving the same final test, the nature of the tests on prior training lists was varied. (For other examples of such designs in investigations of LTL, see Jacoby, 1973; Postman, 1969.) In Experiment 1, a standard yes-no procedure was used on the final common test; on the preceding lists, some subjects were tested by the same method, whereas others were given forced-choice tests. The complementary arrangement was introduced in Experiment 2, with the forced-choice procedure used on the final common test. Failure to observe differential training effects would add force to the conclusion that LTL does not occur in recognition.

The design of Experiment 1 was expanded to determine whether response biases established during training are carried over to a new test situation. Specifically, half of the subjects who were given yes-no tests in the training phase received informative feedback after each choice. Because inexperienced subjects tend to be conservative, it was anticipated that the feedback would encourage them to relax their criterion and to increase the number of their positive responses. If such proved to be the case, the question of interest was whether and to what extent the positive bias would carry over to the critical test task. It was also expected that training with forced-choice tests would be conducive to the establishment of a more lax criterion than would training with standard yes-no tests. Being forced to choose the better of two alternatives each time, subjects might become accustomed to making a positive decision even when they lacked full confidence in it.

\section{EXPERIMENT 1}

\section{Method}

Design. The experimental design comprised five groups. For all subjects, the presentation of a critical list was followed by a yes-no recognition test; the groups differed with respect to the conditions of training that preceded the critical task. The standard practice group went through two training cycles in which the procedure was exactly the same as in the critical phase. A different list was used in each cycle. The training sequence was the same for the informed practice group, except that the subjects were given item-by-item feedback about the correctness of their decisions on the two recognition tests. The forced-choice practice group was also exposed to two training lists but was given a two-alternative forced-choice test of recognition rather than a yes-no test after each list. The frequency-judgment group saw the same lists as the practice groups but, instead of being tested for recognition, was given the task of judging the frequency of usage in the language of both targets and distractors. This group was included to permit an assessment of the interference produced by the processing of verbal materials during the training cycles, without instructions to learn or experience with tests of retention. The control group did not receive any training prior to the presentation of the critical list. However, the control group went through two additional study-test cycles and thus was treated exactly like the standard practice group, yielding additional data for within-subjects analyses of LTL. In the between-subjects analyses of training effects, the first-list scores of the control group and the third-list scores of the standard practice group were the critical values.

Materials. The same three sets of materials were used under all conditions. Each set consisted of a 60-word study list and a test list comprising 60 targets and 60 distractors.

The lists were drawn from a pool of 360 words. This pool was selected from the rank lists of the Kucera-Francis (1967) norms, with two considerations: (1) The range of word frequencies was to be sufficiently wide to make the task of the frequencyjudgment group a reasonable one, and (2) in order to make the tests relatively difficult and thereby to avoid ceiling effects, the preponderance of words was to be sampled from the higher frequency ranks. To satisfy both of these requirements, 48 words were selected from each of pp. 14 of the rank list (Kucera \& Francis, 1967), 24 words from each of pp. 5-8, 12 words from each of pp. 9-12, and 6 words from each of pp. 13-16. Within these constraints, the selection was random, except that function words and proper names were excluded.

The 360 words in the pool were arranged in rank order of frequency and divided into consecutive three-item blocks. Three 120-word sets were then formed by randomly assigning 1 word/block to each set. This procedure ensured that the distributions of frequency values were closely similar in the three sets; the mean frequencies were $137.7,132.7$, and 134.9 , respectively. Each set was in turn partitioned into two 60-word subsets, 
with 1 word/two-item block assigned randomly to each subset. The items in a given subset served as targets for half of the subjects and as distractors for the other half. The six possible sequences of the three sets of materials were used equally often.

All materials were presented in booklets. The 60 words of the study list were arranged on two pages, with each page containing 30 items in a single column. A single random order was constructed for each study list. Whereas study lists were always presented in the same manner, the format of the test booklets varied with the type of test.

In the booklets for the yes-no recognition test, the 120 items ( 60 targets and 60 distractors) were presented on five pages, with 24 words arranged in a single column on each page. The letters " $o$ " (old) and " $n$ " (new) appeared to the right of each word. For a given set of materials, the items were arranged in a single random order, with the restriction that no more than three targets or three distractors appear consecutively. When subjects in the informed practice group were provided feedback during the training cycles, the correct answer (o or $n$ ) appeared to the left of the following word. (As is indicated below, test items were exposed one at a time through a window in a mask.)

In the forced-choice booklets, the 60 target-distractor pairs were presented on three pages, with 20 pairs/page. The pairings were derived from the corresponding yes-no tests as follows. The 120 words on the yes-no test were divided into consecutive blocks of 12 words. Six forced-choice pairs were then constructed from the words in each block, with each pair containing one target and one distractor. The pairs were then placed in the same relative positions as the source words in the yes-no test. The targets appeared as the left-hand and the right-hand members of the pairs equally often, never occupying the same position more than three times in succession.

When the frequency judgments were required, the items were arranged in the same way as on the yes-no test, except that the alternatives "hi" and "lo" (rather than o and n) appeared to the right of each word.

Instructions. The initial instructions described the task requirements during the training cycles. The nature of the recognition test that was to be administered after presentation of the study list was described to the practice groups. The frequency judgment group was informed that they would first be shown a sample of the words to be rated (the study list) and then would make their actual judgments on the entire set of words from which the sample had been drawn (the test list). Their task would be to decide whether the frequency of each word was higher or lower than average in their reading experience. All groups were informed that they would be presented with several sets of materials. Appropriate further instructions were given during each phase of the experiment.

Under the informed practice, forced-choice practice, and frequency-judgment conditions, there was a change in the procedure between the end of the second training cycle and the critical third cycle. In each case, the nature of the change was fully explained, and the necessary new instructions were given prior to the presentation of the critical list.
Procedure. Each page of the study and of the test booklets was covered by a cardboard mask, with a window permitting the exposure of one item at a time. During the study trial, the subjects proceeded through the list at a 2 -sec rate, placing a check mark next to each word and moving the mask down the page to the next item at the sound of a tone signal. (A row of xs appearing in the window served as a signal to change pages.) There was a 2.5 -min interval between the end of each study trial and the corresponding test trial. This interval was filled with a symbolcancellation task and instructions read by the experimenter. The subjects were instructed to respond to each item on the test sheet, underlining the appropriate alternative and guessing if necessary. They proceeded through the test booklet at their own pace, exposing items one at a time through the window of the mask. It was suggested that they spend no more than $3 \mathrm{sec} / \mathrm{item}$, in order to complete the test within $6 \mathrm{~min}$; however, they were given $7 \mathrm{~min}$ to finish. All subjects completed the task within the allotted time.

Subjects. The subjects were undergraduate students who were not necessarily naive to verbal learning experiments but who had not participated in a study of recognition during the same quarter. There were 24 subjects/condition, who were tested in groups of six or fewer, with each group consisting of subjects assigned to the same condition. The order in which conditions were run was determined randomly, with the restriction that each condition occur $n$ times before any one occurred $n+1$ times.

\section{Results and Discussion}

Table 1 summarizes the results of all the recognition tests administered during the experimental sessions. Proportions of misses (M) and false alarms (FA) are listed for the yes-no tests, and proportions of incorrect choices are listed for the forced-choice tests. The values of $d^{\prime}$ are also presented. For the yes-no tests, the measures listed under the heading "M+FA" are the averages of the proportions of the two types of errors." These scores are highly correlated with $d^{\prime}$ values. In the absence of differences in sensitivity, interactions between conditions and types of errors (M vs. FA) are taken to reflect criterion differences (cf. Underwood, 1974).

Training lists. It should be noted at the outset that there were no significant differences between the control group and the standard practice group with respect to accuracy of recognition, distributions of errors, or changes over lists $(\mathrm{Fs}<1.58){ }^{2}$ The two groups, therefore, may be considered as equivalent in ability. Withinsubjects analyses based on their combined results are presented later. In the between-subjects analyses that follow, the data of each group are treated in accordance with each group's designation in the experimental design.

Table 1

Summary of Mean Scores on Successive Recognition Tests (Experiment 1)

\begin{tabular}{|c|c|c|c|c|c|c|c|c|c|c|c|c|}
\hline \multirow[b]{2}{*}{ Condition } & \multicolumn{4}{|c|}{ List 1} & \multicolumn{4}{|c|}{ List 2} & \multicolumn{4}{|c|}{ List 3} \\
\hline & $\mathbf{M}$ & FA & $\mathrm{M}+\mathrm{FA}$ & $\mathrm{d}^{\prime}$ & $\mathbf{M}$ & FA & $\mathrm{M}+\mathrm{FA}$ & $\mathrm{d}^{\prime}$ & M & FA & $\mathrm{M}+\mathrm{FA}$ & $d^{\prime}$ \\
\hline Control & $.350^{*}$ & $.291 *$ & $.320^{*}$ & $1.00^{*}$ & .326 & .278 & .302 & 1.15 & .332 & .281 & .306 & 1.14 \\
\hline Standard Practice & .387 & .289 & .338 & .90 & .385 & .247 & .316 & 1.06 & $.363^{*}$ & $.261 *$ & $.312^{*}$ & $1.20^{*}$ \\
\hline Informed Practice & .326 & .364 & .345 & .86 & .282 & .378 & .330 & .94 & $.300^{*}$ & $.346^{*}$ & $.323 *$ & $1.03^{*}$ \\
\hline Forced-Choice Practice & & & $.262 \dagger$ & .94 & & & $.261 \dagger$ & .97 & $.269^{*}$ & $.383^{*}$ & $.326^{*}$ & $1.01^{*}$ \\
\hline Frequency Judgment & & & & & & & & & $.356^{*}$ & $.323^{*}$ & $.340^{*}$ & $.89^{*}$ \\
\hline
\end{tabular}

* Mean scores on the critical list. Proportion of incorrect choices. 
In the analysis of the results of the recognition tests during the training phase, the standard practice group and the informed practice group, both of which received yes-no tests, are considered together. The proportions of errors (M+FA) were closely similar under the two conditions $(F<1)$. Although the initial level of accuracy was moderate and left substantial room for improvement, the drop in errors between the first and second tests was small and not significant $[F(1,46)=2.43$, $\mathrm{MSe}=.007]$ as was the Condition by List interaction $(\mathrm{F}<1)$. (In the analysis for the $\mathrm{d}^{\prime}$ scores, the corresponding $F$ ratios were 2.77 and less than 1.00 , respectively, $\mathrm{MSe}=.13$.) Although providing informative feedback did not influence the accuracy of recognition, it had a pronounced effect on the distributions of errors. Misses were more frequent than false alarms for the standard practice group, whereas the opposite was true for the informed practice group. The Condition by Type of Error interaction was significant $[F(1,46)=12.94$, MSe $=.032]$. Further analysis showed that the difference between the proportions of misses and of false alarms was significant under each condition. As would be expected if the effects of informative feedback were cumulative, the divergence of the error distributions was greater on the second than on the first test $[F(1,46)=4.14$, MSe $=.007]$ for the Condition by Type of Error by List interaction. As anticipated, therefore, subjects given item-by-item feedback adopted a more lax criterion than did subjects who were given no information about the correctness of their choices. Finally, for the forced-choice practice group, the percentages of errors on the first and the second test were virtually identical $(F<1)$; thus, there was no hint of a practice effect.

In summary, the results obtained in the training phase provide no evidence of significant improvement in discrimination as a function of practice, although there was a trend in that direction for the yes-no tests. Under the latter procedure, response biases were significantly influenced by the conditions of testing, with informative feedback increasing the proportion of both hits and false alarms without affecting the level of discrimination.

Critical test list. The mean scores on the critical test list (the first list of the control group, the first and only test list of the frequency-judgment group, and the third list of the other groups) are marked with asterisks in Table 1. There are no significant differences among the five conditions in either total errors or $\mathrm{d}^{\prime}$ values $(\mathrm{F}<1$ for both measures). Thus, there was no reliable evidence of practice effects on recognition accuracy. Some of the small numerical differences are nevertheless worth noting because they conform to theoretical expectations. The fact that the frequency-judgment group fell below the control group suggests that the processing of the training-list items was a source of interference. The negative component of practice may well be greater when processing is intentional rather than incidental. The slight advantage of the standard practice group over the control group represents a trend in the expected direction. Finally, the scores of the standard practice group were also somewhat higher than those of the groups for which there was a change in the conditions of testing between the training phase and the final common task.

The nature of the tests administered during the training phase did influence substantially the distribution of errors on the critical common test. False alarms were more frequent than misses for the informed practice and forced-choice practice groups, whereas the opposite was true for the other groups. In the case of the informed group, of course, the pattern established during the training phase was carried over to the test list, even though item-by-item feedback was no longer provided. In the case of the group receiving forced-choice practice, the bias toward positive responding emerged without any prior experience with a yes-no recognition procedure. The Condition by Type of Error interaction was significant $[F(4,115)=4.74, \mathrm{MSe}=.019]$. This interaction is accounted for almost entirely by the orthogonal contrast between the informed practice and forced-choice practice groups, on the one hand, and the remaining three groups, on the other $[F(1,115)=15.89]$. It is interesting to note that the false alarm rate was relatively high for the frequency-judgment group; the interference produced by exposure to the training lists would be expected to influence primarily the probability of false positive responses.

Within-group analyses. The combined data of the standard practice and control groups, which were treated alike throughout, were used in a detailed examination of performance measures on the successive tests. The combined groups provide a substantial sample for withinsubjects analyses, which should be maximally sensitive to any systematic trends as a function of practice. Before describing the attempts to identify such trends, we note that a within-subjects analysis also failed to yield statistically acceptable evidence for improvements in recognition performance across lists $[F(2,94)=1.40$ for errors and 2.56 for $\mathrm{d}^{\prime}$ scores, $\mathrm{MSe}=.01$ and .25 , respectively 1 .

The main question of interest was whether and to what extent interference effects developed within and across tests. With this question in mind, the proportions of misses and false alarms given on the five successive pages of the test booklets were examined. Each page contained 12 targets and 12 distractors. As was noted earlier, there were six different test forms, which were used equally often in the three practice cycles.

False alarm rates are considered first because they bear directly on the buildup of interference as a function of practice. As subjects proceed through a test, it should become increasingly likely that distractors appear familiar because they share features with items seen previously, notably other distractors (cf. Underwood, 1971). If the resulting interference is cumulative, false alarm rates should increase progressively, not only within tests, but also from one test to the next. Even if the proactive 
effects of prior lists are relatively weak, they might summate with the interferences developed during a test. If so, the rises in false alarms should become more pronounced as practice continues.

As can be seen in Table 2, the false alarm rate increased between the first and second segment on each test. Apart from one deviant entry (the fourth segment of the second test), subsequent changes were relatively minor. Numerically, the rate was always highest for the last segment. The implication is that the interference produced by exposure to the test items built up rapidly at first and grew slowly, if at all, thereafter. The interference clearly did not carry over from one test to the next: The functions for the successive lists overlapped closely. A linear trend analysis showed that the rise in false alarm rates over segments was significant overall $[\mathrm{F}(1,47)=20.58$, MSe $=.016]$, with no reliable differences among the lists $[\mathrm{F}(2,94)=1.35, \mathrm{MSe}=.017]$.

To determine whether susceptibility to test-produced interference influenced overall recognition accuracy, the subjects' total scores in the linear trend analysis (weighted sums of proportions for the five segments) were correlated with their mean $d^{\prime}$ scores. The correlation was close to zero $(r=.097)$. It appears, therefore, that good learners are as vulnerable to test-produced interference as are poor ones.

Misses are considered next. Here rises across segments are to be expected on the basis of "normal forgetting" alone, in particular progressive increases in retroactive effects. Specifically, presentation of the targets in a random sequence ${ }^{3}$ necessarily produces a correlation between test order and length of retention interval: The later a target appears, the longer the filled delay following its presentation is likely to be. As indexed by the numbers of intervening study and test items, the average retention intervals for the targets on the five successive pages of the test booklets were 40.6, 88.9, 116.4, and 137.0 , respectively. (The values are quite similar for each of the six test forms.) Nevertheless, it appeared possible that proactive interference from prior lists would produce shifts in the test-order functions such as those considered above for false alarms.

The expected increases occurred. Except for one reversal on the first test, they did not emerge before the fourth segment. The fact that misses increased so slowly may be viewed as consistent with Schulman's (1971)

Table 2

Mean Proportions of Misses and False Alarms on Successive Pages of Tests (Standard Practice, Experiment 1)

\begin{tabular}{cccccccc}
\hline & \multicolumn{3}{c}{ Misses } & & \multicolumn{3}{c}{ False Alarms } \\
\cline { 2 - 4 } \cline { 6 - 8 } Page & List 1 & List 2 & List 3 & & List 1 & List 2 & List 3 \\
\hline 1 & .321 & .342 & .340 & .215 & .233 & .210 \\
2 & .370 & .342 & .339 & & .311 & .287 & .288 \\
3 & .325 & .328 & .332 & & .300 & .281 & .280 \\
4 & .412 & .390 & .408 & & .286 & .240 & .278 \\
5 & .413 & .375 & .319 & .336 & .295 & .297 \\
\hline
\end{tabular}

conclusion that recognition performance is relatively insensitive to retroactive interference from intervening items. An unexpected finding, which complicates the picture, is the sharp decline in misses in the final seg. ment of the third test. The finding appears to be a reliable one; it remains clearly in evidence when the two groups that were given standard practice are considered separately. One may speculate that subjects became less conservative as they came to the end of the session; there was also an increase in false alarms, although not an unusually large one. It may be worth investigating whether "end spurts" of positive responding occur in other situations. Apart from the terminal drop, there were no systematic changes across tests, and certainly there was no evidence of cumulative proactive interference.

In the analysis of changes across segments, the overall linear trend was found to be significant $[\mathrm{F}(1,47)=8.96, \mathrm{MSe}=.024]$. The differences among lists were not reliable $[F(2,94)=2.33, \mathrm{MSe}=.02]$. Since the terminal drop in misses was an unexpected and interesting finding, the differences between the fourth and fifth segments were examined in a post hoc analysis. For these difference scores, the shift between the first and the third test was significant $[F(1,47)=8.32$, $\mathrm{MSe}=.048]$. Finally, the correlation between the linear trend scores and the average $d^{\prime}$ scores was close to zero, as before $(r=-.057)$.

It should be noted that the present results differ somewhat from those reported by McCormack and Swenson (1972), who found no significant changes in hit rates either within or between lists. The false alarm rates, on the other hand, rose within lists and also increased slightly across lists. The discrepancies may reasonably be attributed to differences between the materials used in the two experiments. The lists in the investigation of McCormack and Swenson were quite short (21 study items and 42 test items) and homogeneous with respect to word frequency (either all high frequency or all low frequency). Thus, the variations in retention interval associated with test order were too small to produce appreciable changes in hit rates; some minor declines are, in fact, apparent in their data. The homogeneity of the lists probably accounts for the small, and not entirely consistent, increases in false alarms across lists.

\section{EXPERIMENT 2}

The purpose of Experiment 2 was to obtain additional data on practice effects in recognition, with a forcedchoice procedure used on the final critical test. The design was limited to two groups. For the forced-choice practice group, the conditions of testing during the training phase and for the final list remained the same. For the standard practice group, yes-no tests on the first two lists were followed by a forced-choice test on the final list. 


\section{Method}

Except for the changes in the sequence of tests, the materials and procedure were exactly the same as those for the corresponding groups in Experiment 1. There were 24 subjects in each group, drawn from the same pool as before. No more than six subjects were tested at the same time.

\section{Results and Discussion}

The measures of performance on the three successive tests are summarized in Table 3 .

Training lists. For the forced-choice practice group, the proportion of errors dropped slightly between the first and the second lists $(F<1)$. The results for the standard practice group paralleled those obtained in Experiment 1. Misses were again more frequent than false alarms $[F(1,23)=11.94, \mathrm{MSe}=.008]$. The small increase in accuracy on the second test was not significant $\left[F(1,23)=1.50\right.$ for errors and 1.40 for $\mathrm{d}^{\prime}$ scores, $\mathrm{MSe}=.034$ and .15 , respectively]. Under neither condition, therefore, was there statistically acceptable evidence of practice effects.

Critical test lists. The proportions of errors on the critical test list were nearly the same under the two conditions $(F<1)$. As was found for the terminal yes-no test in Experiment 1, therefore, there were no differential training effects. The scores on the critical list were quite similar to the first-list scores of the forced-choice practice group in Experiment 1 (see Table 1). This comparison confirmed the absence of practice gains.

Within-group analyses. The data of the forced-choice practice group, for which the conditions of testing remained the same, were used in within-subjects analyses of the measure of performance on the three successive tests. The results can be summarized briefly: (1) There were still no reliable practice effects when all three lists were considered $(F<1)$. (2) As was true for the standard practice groups in Experiment 1, there were within-test increases in the error rates. In the present case, the proportions of errors on the three pages of the forcedchoice booklets, each containing 20 test pairs, were compared. The relevant data are presented in Table 4. There was evidence of progressive increases on each test. The overall linear trend was significant $[F(1,23)=18.23$, $\mathrm{MSe}=.01 \mathrm{]}$, with no reliable differences among the lists $(\mathrm{F}<1)$. It is not possible to say, of course, to what extent variations in the length of the retention interval and test-produced interference contributed to this effect. Again, there was no evidence of cumulative interlist interference. (3) The correlation between the linear
Table 4

Mean Proportions of Errors on Successive Pages of Tests (Forced-Choice Practice, Experiment 2)

\begin{tabular}{cccc}
\multicolumn{4}{c}{ (Forced-Choice Practice, Experiment 2) } \\
\hline Test Page & List 1 & List 2 & List 3 \\
\hline 1 & .240 & .223 & .204 \\
2 & .273 & .256 & .269 \\
3 & .310 & .285 & .288 \\
\hline
\end{tabular}

trend scores and mean $\mathrm{d}^{\prime}$ scores was not significant, but it was in the expected direction $(r=-.268)$.

\section{GENERAL DISCUSSION}

The main results of the present study may be summarized as follows: Recognition performance failed to improve significantly as a function of practice. Numerically, there were some slight but consistent gains on the yes-no tests, but not on the forced-choice tests. When the methods of measurement during the training phase and on the final critical test were the same, recognition accuracy was no higher than when there was a change in procedure. The conditions of training did, however, influence the criterion adopted by subjects on a final yes-no test. Informative feedback and experience with a forced-choice procedure in the training cycles were both conducive to the setting of relatively lax criteria. In both cases, subjects were encouraged to respond positively to items about which they were less than certain.

The absence of reliable practice gains in recognition assumes importance in light of the common occurrence of LTL effects for memory tasks requiring the retrieval of target items. Although this contrast deserves emphasis, some qualifications must be added. The bulk of the evidence for LTL in recall tasks comes from experiments in which multiple-trial procedures were used, in particular serial and paired associate learning. Task-specific skills may be more likely to develop when training lists are tested more than once, so that subjects have an opportunity to modify their strategies with reference to a specific set of materials. This conjecture receives some support from the fact that performance has been found to improve with practice in multiple-trial free recall (Dallett, 1963; Mayhew, 1967; Postman, Burns, \& Hasher, 1970; Rosner, 1970; Tulving, McNulty, \& Ozier, 1965), but not in single-trial free recall (Dallett, 1963; Jacoby, 1973; Raymond, 1969). However, Jacoby (1973) did observe practice gains in single-trial cued

Table 3

Mean Soores on Successive Tests in Experiment 2

\begin{tabular}{|c|c|c|c|c|c|c|c|c|c|c|}
\hline \multirow[b]{2}{*}{ Condition } & \multicolumn{4}{|c|}{ List 1} & \multicolumn{4}{|c|}{ List 2} & \multicolumn{2}{|c|}{ List 3} \\
\hline & $\mathbf{M}$ & FA & $\mathrm{M}+\mathrm{FA}$ & $d^{\prime}$ & $\mathbf{M}$ & FA & $\mathrm{M}+\mathrm{FA}$ & $\mathrm{d}^{\prime}$ & Errors & $\mathrm{d}^{\prime}$ \\
\hline $\begin{array}{l}\text { Forced-Choice Practice } \\
\text { Standard Practice }\end{array}$ & .410 & .257 & $\begin{array}{l}.274^{*} \\
.334\end{array}$ & $\begin{array}{l}.90 \\
.97\end{array}$ & .365 & .258 & $\begin{array}{l}.255^{*} \\
.312\end{array}$ & $\begin{array}{l}1.05 \\
1.11\end{array}$ & $\begin{array}{l}.254 \\
.242\end{array}$ & $\begin{array}{l}1.08 \\
1.05\end{array}$ \\
\hline
\end{tabular}

*Proportion of incorrect choices. 
recall. In addition, LTL has been shown to occur in multiple-trial verbal discrimination learning, a task that does not require response retrieval and has been viewed as being closely related to recognition in depending on the retention of frequency information (Underwood, Shaughnessy, \& Zimmerman, 1972).

Although a full consideration of the evidence on LTL introduces ambiguity into the picture, the results obtained in investigations of test-expectancy effects reinforce the conclusion that recall is more likely to benefit from task-specific training than is recognition (for a recent review of the relevant studies, see Neely \& Balota, 1981). A common procedure for inducing test expectancies is to have subjects go through several study-test cycles in which one or the other method of measuring retention (i.e, either recall or recognition) is used each time. ${ }^{4}$ In the final phase, the method of measurement either remains the same or changes, respectively confirming or violating the subjects' expectancies. When the critical final test requires recall, subjects expecting such a test typically perform better than do those expecting recognition. By contrast, the complementary expectancy effects on final tests of recognition have usually failed to materialize; in fact, recent well controlled studies have shown that individuals anticipating a test of recall surpass those anticipating a test of recognition (Balota \& Neely, 1980; Neely \& Balota, 1981). A test-expectancy effect, of course, is not contingent on LTL gains during the preceding trials; it occurs to the extent that the subjects' encoding operations are more appropriate to the test that they actually receive than to the alternative one. Nevertheless, the asymmetry of the observed effects implies that subjects do not command, and cannot readily refine, encoding strategies that might serve to improve their recognition accuracy. ${ }^{5}$ The question of why anticipation of a test of recall serves to improve recognition need not concern us further here. Suffice it to note briefly the hypothesis proposed by Neely and Balota (1981) that subjects expecting a test of recall attend to more different meanings of target words than do subjects expecting a test of recognition.

We found no evidence to support the assumption that cumulative interlist interference masks LTL effects in recognition. The only observation that pointed in this direction was the relatively high false alarm rate under the frequency-judgment condition in Experiment 1. Since this increase in false positive responses did not approach significance, no undue importance can be attached to it. The within-test rises in false alarms show that exposure to distractors was, indeed, a source of interference. There was no indication, however, that such interference was carried over from one test to the next. Although it does not help to throw new light on the absence of practice gains, this finding is of considerable interest in its own right. Commenting on the very small betweenlist increases in false alarm rates they observed, McCormack and Swenson (1972, p. 77) suggested that the interval between successive lists "appears to allow for a release from interference." It is far from clear, of course, why the release occurs when there are no systematic changes in either materials or the conditions of list presentation and testing. Somehow, the interference is tied to the context of a particular test. This problem may be well worth pursuing.

We are reluctant at this point to subscribe to the notion that storage of the occurrence information requisite for recognition is an automatic process. Hasher and Chromiak (1977) concluded that frequency tagging is automatic after finding neither developmental changes nor practice effects for frequency judgments. Performance on recognition tests seems to meet the criteria they used for inferring automaticity. There is an apparent absence of practice gains in adults, and the indications are that recognition memory is well developed in young children for pictures (cf. Brown \& Campione, 1972) as well as for words (Naus et al., 1977). The apparent difficulty with the hypothesis of automatic processing is that it rests on null results. Level of performance may reach an asymptote at an early age because the requisite skills are acquired rapidly, not because they come into play automatically. Practice effects may fail to materialize because further improvement is difficult or because the conditions of training are less than optimal.

At this point, it appears reasonable to attribute the absence of significant LTL effects in recognition to the difficulty of identifying test-appropriate strategies. As discussed earlier, lack of full information about the characteristics of the distractors is probably an important source of this difficulty. If so, practice effects might develop gradually and slowly as subjects come to focus on target attributes most likely to prove useful for discriminative decisions. The small and unreliable improvements observed under several conditions in this study may be viewed in this light. The possibility cannot be ruled out that LTL effects could be enhanced by special instructional procedures. As the present results and those of earlier investigations show, however, subjects left to their own devices are likely to register minor practice gains at best. By contrast, response bias, which is under the learner's immediate control, is readily modified by prior training.

\section{REFERENCES}

Balota, D. A., \& Neely, J. H. Test-expectancy and wordfrequency effects in recall and recognition. Journal of Experimental Psychology: Human Learning and Memory, 1980, 6, 576-587.

Brown, A. L., \& Campione, J. C. Recognition memory for perceptually similar pictures in preschool children. Journal of Experimental Psychology, 1972, 95, 55-62.

CAREY, S. T., \& LockhaRT, R. S. Encoding differences in recognition and recall. Memory \& Cognition, 1973, 1, 297-300.

Connor, J. M. Effects of organization and expectancy on recall and recognition. Memory \& Cognition, 1977, 5, 315-318.

Dallett, K. M. Practice effects in free and ordered recall. Journal of Experimental Psychology, 1963, 66, 65-71.

Hasher, L., \& Chromink, W. The processing of frequency information: An automatic mechanism? Journal of Verbal Learning and Verbal Behavior, 1977, 16, 173-184.

$\mathrm{J}_{\mathrm{ACOBY}}, \mathrm{L}$. L. Test appropriate strategies in retention of cate- 
gorized lists. Journal of Verbal Learning and Verbal Behavior, 1973, 12, 675-682.

KuČERA, J., \& Francis, W. Conceptual analysis of present-day American English. Providence, R.I: Brown University Press, 1967.

MAYHEW, A. J. Interlist changes in subjective organization during free-recall learning. Journal of Experimental Psychology, 1967, 74, 425-430.

McCormack, P. D., \& Swenson, A. L. Recognition memory for common and rare words. Journal of Experimental Psychology, 1972, 95, 72-77.

Naus, M. J., Ornstein, P. A., \& Kresthool, K. Developmental differences in recall and recognition: The relationship between rehearsal and memory as test expectation changes. Journal of Experimental Child Psychology, 1977, 23, 252-265.

NeEly, J. H., \& Balota, D. A. Test-expectancy and semanticorganization effects in recall and recognition. Memory \& Cognition, 1981, 9, 283-300.

Postman, L. Experimental analysis of learning to learn. In G. H. Bower \& J. T. Spence (Eds.), The psychology of learning and motivation (Vol. 3). New York: Academic Press, 1969.

Postman, L. Transfer, interference and forgetting. In J. W. Kling \& L. A. Riggs (Eds.), Experimental psychology. New York: Holt, Rinehart \& Winston, 1971.

Postman, L., Burns, S., \& Hasher, L. Studies of learning to learn: $X$. Nonspecific transfer effects in free-recall learning. Journal of Verbal Learning and Verbal Behavior, 1970, 9, 707. 715.

RAYmond, B. Short-term storage and long-term storage in free recall. Journal of Verbal Learning and Verbal Behavior, 1969, 8, 567-574.

ROSNER, S. R. The effects of presentation and recall trials on organization in multitrial free recall. Journal of Verbal Learning and Verbal Behavior, 1970, 9, 69-74.

Schulman, A. I. Recognition memory for targets from a scanned word list. British Journal of Psychology, 1971, 62, 335-346.

Tulving, E., McNulty, J. A., \& Ozier, M. Vividness of words and learning to learn in free-recall learning. Canadian Journal of Psychology, 1965, 19, 242-252.

TVE Rsky, B. Encoding processes in recognition and recall. Cognitive Psychology, 1973, 5, 275-287.

TVERSKY, B. Eye fixations in prediction of recognition and recall. Memory \& Cognition, 1974, 2, 275-278.

UNDERWOOD, B. J. Recognition memory. In H. H. Kendler \& J. T. Spence (Eds.), Essays in neobehaviorism. New York: Appleton-Century-Crofts, 1971.

UNDE RWOOD, B. J. The role of the association in recognition memory. Journal of Experimental Psychology Monograph, 1974, 105, 917-939.

Underwood, B. J., Shaughnessy, J. J., \& Zimmerman, J. Learning-to-learn verbal-discrimination lists. Journal of Verbal Learning and Verbal Behavior, 1972, 11, 96-104.

\section{NOTES}

1. If one thinks in terms of separate "yes" and "no" tests, the observed proportion of the joint event (M, FA) roughly corresponds to an error on a forced-choice test. I am grateful to R. A. Bjork for calling this point to my attention.

2. The level of significance was set at $p<.05$.

3. Targets were presented in a random, rather than in a firstin, first-out order to discourage the development of serial learning strategies.

4. Several of the experiments cited above, which failed to yield evidence of practice gains, were studies of the effects of test expectancy.

5. An exception is Tversky's $(1973,1974)$ finding that testappropriate instructions prior to study improve recognition of pictures. This result appears to be specific to pictorial materials, with which subjects expecting recognition can focus on distinctive details of the visual stimuli.

(Received for publication January 19, 1982; revision accepted April 5, 1982.) 